\title{
Accurate Position Estimation in Switched Reluctance Motor With Smooth Starting
}

\author{
Debiprasad Panda, Student member IEEE, and V. Ramanarayanan \\ Department of Electrical Engineering, \\ Indian Institute of Science, Bangalore 560012, India \\ email: dpanda@ee.iisc.ernet.in, vram@ee.iisc.ernet.in
}

\begin{abstract}
This paper presents a novel method of position estimation for Switched Reluctance (SR) Motor. The method is suitable from starting to full speed. It ensures smooth starting without initial hesitation. The method further proposes a better position estimating algorithm incorporating corrections for eddy current and mutual inductance effects. The algorithm is better suited in a digital control platform for its realisation. In the present work, a Texas Instruments make DSP (TMS320c50) is used for the position estimation as well as control of the SR motor.
\end{abstract}

\section{INTRODUCTION}

Switched Reluctance motor with its inherent simplicity and fault-tolerant capability is an attractive option for variable speed applications. The position information requirement is a limitations of this motor. The shaft position sensors are normally used for this purpose. These sensors reduce the reliability of the drive. Efforts are on to replace the position sensor with suitable estimation technique. To this effect, a number of sensorless algorithm with different degree of success is published. Unfortunately most of the existing sensorless schemes do not cover the starting problem. Few sensorless starting methods are suggested by different authors and each of them has their own limitation.

In this paper, an overview of the different existing sensorless starting methods and their shortcomings are explained and finally, a novel starting method is proposed. An improved position estimator incorporating the corrections due to eddy current and mutual fluxes is realised.

\section{POSITION ESTIMATION THROUGH FLUX-CURRENT METHOD}

Among the existing sensorless methods, those based on the flux-linkage characteristics have become popular [1-3]. Figure 1 shows the block schematic of the basis of position estimation. Flux-linkage of any phase is computed from Eq. (1).

$$
\Psi=\int(V-R i) d t
$$

Pre-computed $\psi$ - $\mathrm{i}$ characteristics as shown in Fig. 2 is stored in a look-up table to compute position from the measured phase voltages and currents. From the measured voltage and current, flux-linkage of a phase is computed from (1). With known flux-linkage and current, position can be looked-up from the stored data. There are several important issues involved in the position estimation method outlined above. These are listed as follows.

The $\psi$ - i characteristics are pre-computed. The precomputed characteristics are obtained by tests done under static conditions [4]. These static tests do not take into account the non-idealities associated with the dynamic conditions under which the machine is operating. Such non idealities are related to two aspects-

(i) The eddy current effects in dynamic condition may influence the $\psi-i$ characteristics of the SR motor. These influences may introduce an error in the estimated position. The eddy current effects and its remedy are reported in an earlier publication [3]. (ii) The static $\psi-i$ characteristics does not take into effect the mutual coupling between the various phases during dynamic operation. It is shown that

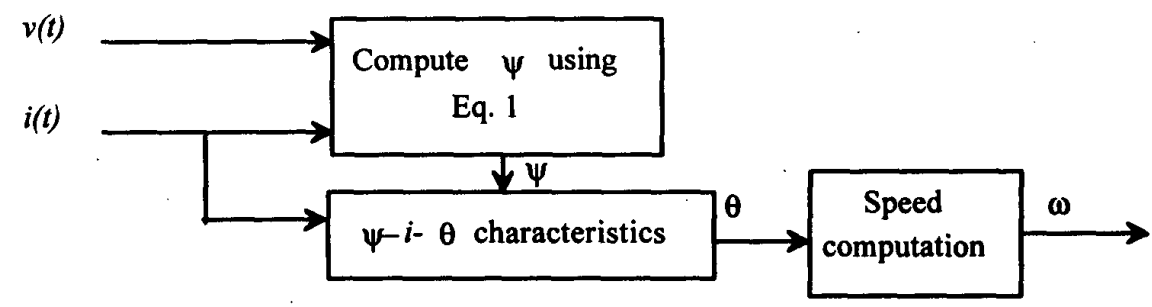

Fig. 1 Schematic of the Position Estimation Method 


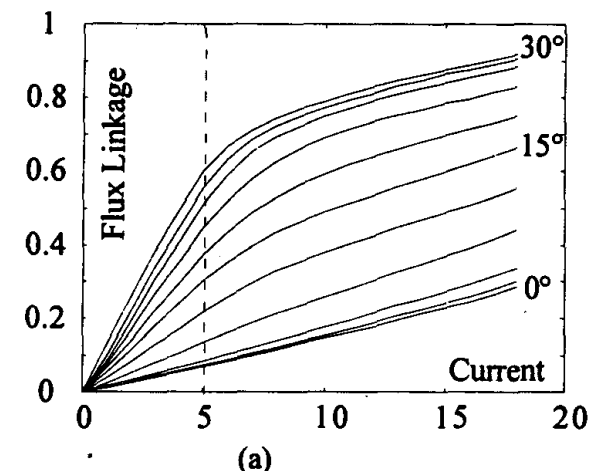

(a)

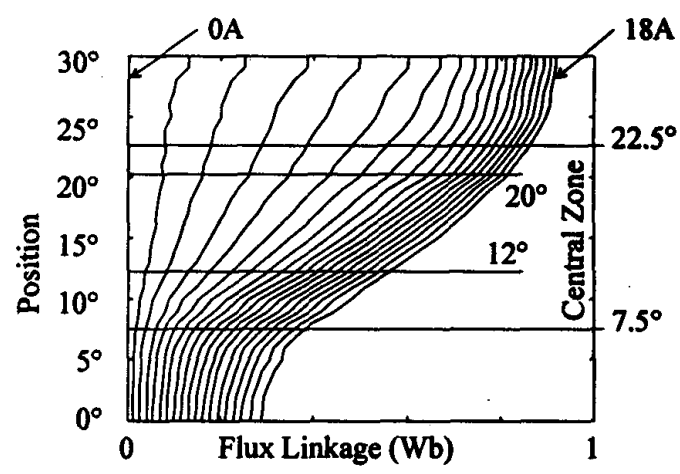

(b)

Fig. 2 Flux-Linkage Characteristics

mutual fluxes can introduce errors in position estimate as much as $\pm 3^{\circ}[2]$.

Most position estimation methods do not cover starting condition. It is known that, SR motors with sensorless operation suffer from starting problem. In this paper, a novel starting algorithm is proposed.

\section{SENSORLESS STARTING OF SR MOTOR}

During standstill, the rotor position is unknown. For smooth starting of the drive, the relative position of the phases should be detected and proper phase should be chosen for excitation for driving the motor in the required direction. Excitation to any arbitrary phase will not result smooth starting. In literature, a number of sensorless algorithm for SR motor drive are suggested. Unfortunately, most of the existing schemes discuss the position estimation only during running condition. Without successful starting, the sensorless operation only in running condition does not make much sense. Proper algorithm is desired which will give better position estimate during running as well as in starting. A short review of the starting methods are explained below.

\section{A. Feedforward open loop method}

The motor is controlled feedforward in open loop as a stepper motor [5]. A train of pulses with initial frequency is applied to each phase of the motor windings in a sequence according to the direction of rotation. The dwell angle (difference between Toff and Ton angle) [6] is kept fixed and each phase pulse train is phase shifted by $15^{\circ}$. The frequency is increased linearly, and it is assumed that the rotor follows it. Lopez et al [5] has demonstrated this method in connection to their current gradient sensorless method. According to the above authors, the method is neither efficient nor optimal, but it permits a reliable start even under load.

\section{B. Locking the rotor along a particular phase axis}

By exciting a particular phase of the motor for sufficient amount of time, the rotor may be aligned along its axis [7]. Once the rotor is aligned, the position of all the phases with respect to the rotor is known and by appropriate switching logic the motor can start suitably. We have observed that by exciting only one phase, the rotor may not get aligned to the required axis. The inductance profile of the test motor are designed in such a way that near both aligned and unaligned region there is a no-torque zone, when even under excitation the rotor will not experience any torque (c.f. Fig. 3b). Hence, originally if the rotor lies in the no-torque zone near unaligned region, it will not be aligned even with excitation. In that situation, one phase excitation may not be sufficient for alignment of the rotor. Two phases are used for reliable alignment. The major disadvantage of this method is that the motor may move backwards before actual starting commerices.

\section{Flux-computation technique}

During standstill, a pulse voltage may be injected to any phase and flux-linkage may be computed through the integration of voltage equation. From the measured current and computed flux, position at starting may be obtained from the stored flux-linkage characteristics in the same way as the running condition. Flux-linkage characteristics being symmetric along the aligned axis, it is not possible to estimate the absolute position of the rotor through flux-current method by single phase excitation. Theoretically, by giving excitation to two phases the exact rotor position of the different phases can be detected. In order to obtain accurate estimate of position, phases are to be excited for sufficient time so that sufficient flux builds up, otherwise at low flux and low current the estimation results will be erroneous (c.f. Fig. 2). Besides, if the particular phase is near unaligned or aligned region, the estimated position will be error prone. The main difficulty with this method is that before the actual start, the rotor may move backward and a starting hesitation is observed. Hence, in this two phase excitation method special care is to be taken for avoiding such hesitation. 


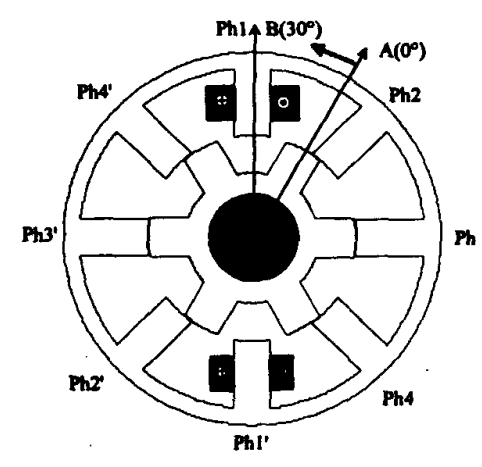

Fig. 3a Cross Section of an $8 / 6$ pole, 4-phase SR motor (only one phase is shown with winding for clarity of the figure).

\section{Four-phase Excitation method}

Position can be detected most reliably at starting by giving test excitation to all the 4-phases simultaneously. By exciting all the phases of motor upto a moderate value of flux and current, there will be no appreciable rotor movement. This aspect has been addressed by a recent paper by $\mathrm{Bu}$ and $\mathrm{Xu}$ [8]. Same approach as theirs with certain modification has been followed in the present work. This method with both discrete as well as continuous estimation methods is demonstrated with experimental results. This proposed method is explained in the following section.

\section{E Proposed 4-phase excitation method for Starting}

The magnetic characteristics of the motor with flux-linkage as a function of current and position is shown in Fig. 2. It is observed that the slope of each of the curves are almost constant upto certain level of current. For the test motor this current level is around 5A (c.f. Fig. 2). In standstill condition, the votage Eqn. of a phase will be as follows-

$$
\begin{aligned}
& v=R i+\frac{\partial \psi}{\partial i} \frac{d i}{d t}+\frac{\partial \psi}{\partial \theta} \frac{d \theta}{d t}(\approx 0) \\
& =R i+l \frac{d i}{d t}
\end{aligned}
$$

It may be seen that upto a certain current level, inductance $l$ of a phase remains constant for a particular position and Fig. 3b shows that with linearised operation the inductance increases proportionately with position. Thus, if the excitation is restricted to the linear zone of the magnetic characteristics and all the phases are excited from a same source voltage for same duration of time, then the rate of rise of current of the phases will be inversely proportional to their linear inductance. Following this explanation, the current of the phase near unaligned region will be more than the phase at aligned position. The phases in the intermediate positions will carry currents in between these two extreme values.

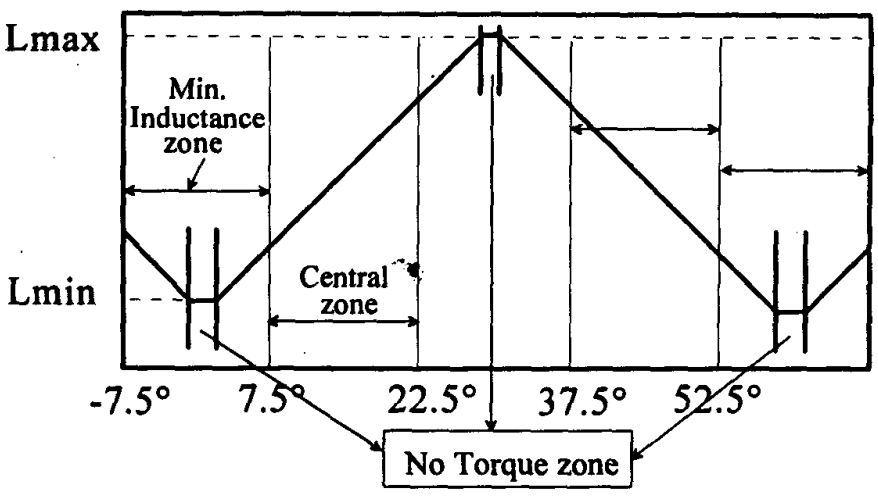

Fig .3b Idealised Inductance of an individual phase with respect to its rotor position

Therefore, in standstill, the rate of rise of current indicates the relative position of the phases.

For an $8 / 6$ pole SR motor, the relative displacement between the phases are $15^{\circ}$. Thus one phase is available within any $15^{\circ}$ zone. Among these, the phases in central zone $\left(7.5^{\circ}\right.$ to $22.5^{\circ}$ or $37.5^{\circ}$ to $\left.52.5^{\circ}\right)$ produces maximum torque for a given current. Depending on the direction of rotation, either of these two phases (which are in the central zone), are to be chosen as the active torque producing phase and this active phase is suitable for position estimation as well.

In order to detect the active phase, $\mathrm{Bu}$ and $\mathrm{Xu}[8]$ recently have suggested a method with all four phase excitation. In their method, all the phases are excited simultaneously with same voltage. With this condition, current in all the phases are monitored after a predetermined time (say $t 1$ ). At the time instant $t 1$, the phase carrying maximum current is detected. The phase carrying maximum current will certainly have minimum inductance and must be stationed near unaligned position. The immediate phase prior to it in the sequence (for anticlockwise, rotation $\mathrm{Ph} 1-\mathrm{Ph} 2-\mathrm{Ph} 3-\mathrm{Ph} 4-\mathrm{Ph} 1$ and for clockwise rotation $\mathrm{Ph} 4-\mathrm{Ph} 3-\mathrm{Ph} 2-\mathrm{Ph} 1-\mathrm{Ph} 4$ is the sequence, c.f. Fig. 3a) will be in the central zone $\left(+7.5^{\circ}\right.$ to $+22.5^{\circ}$ region, c.f. Fig. $\left.3 \mathrm{~b}\right)$. Thus the phase in central zone is detecteo and estimation is continued with that phase till the next phase enters the central zone. This can be explained with the following example.

Suppose, phasel (Phl) is carrying maximum current. Hence $\mathrm{Phl}$ is located nearest to the unaligned position $\left(-7.5^{\circ}\right.$ to $+7.5^{\circ}$ ) (c.f. Fig. 3 b). In this case, for clockwise rotation, $\mathrm{Ph} 4$ must be within $+7.5^{\circ}$ to $+22.5^{\circ}$ region in the positive inductance slope which is referred as active phase. On excitation, $\mathrm{Ph} 4$ will produce positive torque and the motor will start rotating in the desired direction. Following the similar explanation, for anticlockwise rotation $\mathrm{Ph} 2$ will be excited first. 
The problem in this method is that the time instant $t 1$ has to be chosen with sufficient care so that the current in any of the phases do not go beyond a certain limit. At higher currents, the magnetic core saturates and inductance no longer remains linear. In such case, the incremental inductance of aligned region may be less than or equivalent to that at unaligned region. Rate of rise of current in this condition near aligned region may be even more than that of at unaligned region. Hence, by allowing the excitation for excess time, the current may rise high enough to saturate the core and no logical conclusion may be made regarding the relative position between the different phases by monitoring the current magnitude of the phases. Thus, there must be an upper limit of $t 1$ for a given motor. On the other hand, time $\mathrm{t} l$ has to be sufficiently high so that the difference of currents in the different phases are distinguishable. Hence, there must be a lower limit on $\mathrm{tl}$ as well. Thus choice of $\mathrm{t} l$ is not very straightforward and it will differ from machine to machine.

In order to avoid such ambiguity, an alternate method is proposed here. From the magnetic characteristics, one can easily detect the current level below which the flux-linkage characteristics of the machine is linear irrespective of position. Such value of current is referred as the threshold. With a known threshold, all the phases are excited simultaneously and the phase currents are monitored at every sampling time. Whenever the current in any of the phases exceed the threshold, all the phases except the one just ahead in sequence of this phase, are switched off. The phase just ahead in sequence of this maximum current carrying phase is referred as the active phase. For the test motor, the threshold current limit is chosen as $5 \mathrm{~A}$ (almost one third of the rated current). There is a theoretical possibility of two phases attaining the threshold current limit at the same time. This possibility may arise when two consecutive phases rest originally at $-7.5^{\circ}$ and $+7.5^{\circ}$ position respectively. In that case, the phase ahead in the sequence is chosen as the active phase. Following this method, the excessive current in the phases can be avoided and the detection of initial active phase can also be made without ambiguity. In this method, no off-line computation of time $t 1$ is needed.

\section{POSITION ESTIMATION ALGORITHM}

The position estimation algorithm reported in this paper is a composite method incorporating the following features.

On starting, test excitation is employed to select the appropriate phases to be excited. Direct control of the current injected during starting ensures test pulse in the non-saturated zone of $\psi-i$ characteristics. Following this approach smooth starting is possible.

On running, the algorithm incorporates the following corrections.

(i) Appropriate phase is chosen for estimation. Whenever, a particular phase is within $7.5^{\circ}$ to $22.5^{\circ}$ (central

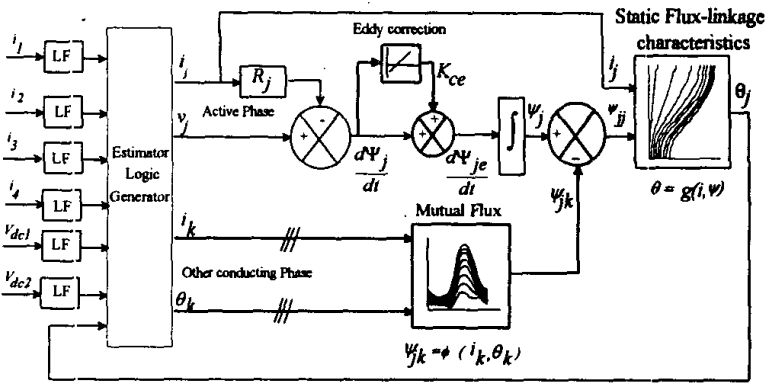

Fig. 4 Block diagram of Continuous Position Estimator using Flux/current Method including eddy-current and mutual flux correction (shown in blod line).

zone, c.f. Fig. 2), it is called the active phase and that phase is chosen for position estimation during that period. In this zone, the sensitivity of position to flux is minimum. Thus any error due to measurement of current and flux will not introduce much error in terms of estimated position.

(ii) The nature and quantum of corrections required to account for eddy current effects and mutual coupling effects are obtained through off-line measurements. Such correcticns are applied to the computation of flux in order to make it compatible with the stored static $\psi-i$ characteristics. The measured results of eddy current and mutual flux are explained details in [2, 3].

\section{POSITION ESTIMATOR REALISATION}

The block diagram of the position estimator following the above method is shown in Fig. 4. Six analog signals (the four phase currents and two dc link voltages) are taken as inputs through ADC. All analog signals are processed through software low pass filter (LF) to attenuate the switching, and high frequency noises. The estimator logic generation block decides on the active phase. Eddy-current effect is decided by the rate of change of flux-linkage in the active phase. Thus in this work, the eddy current correction term, $K_{C^{2}}$, is made a function of $\frac{d \psi_{j}}{d t}$. The corrected gradient of flux is shown in the figure as $\frac{d \psi_{j e}}{d t}$. The computed flux obtained through the integration is further corrected for mutual flux due to the other conducting phases. This corrected flux may be treated same as the static flux-linkage characteristics. From the corrected flux $\psi_{j j}$ and the active phase current $i_{j}$, position is looked-up from the stored static flux-linkage characteristics. From the figure, it may be seen that three look-up tables are searched at every sample time, namely - (i) eddy current correction, (ii) mutual flux and (iii) position fiom static flux-linkage.

\section{CONTROLLER REALISATION}

Apart from the above estimator, the control of SR motor involves a number of operations such as speed computation, execution of speed and current controller, 


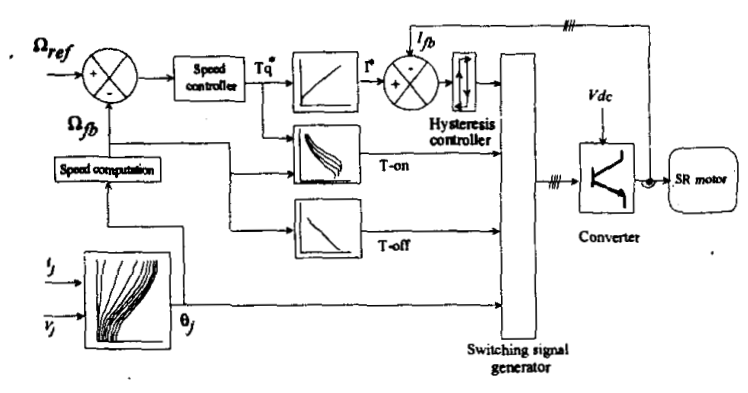

Fig. 5 Controller block diagram including Estimator

execution of torque to current conversion, T-on and T-off angle selection (Turn-on and Turn-off angle of each phase) and final switching logic generation etc. Besides the estimator, all these jobs are to be carried out once in every sample period. The block diagram of complete controller is shown in Fig. 5. A PI controller serves as speed controller and the current control is through hysteresis.

The execution of the control scheme (including the estimator) as discussed above is time critical. Digital controller is ideal choice for realising such controllers. In this work, a Texas Instruments make, 16-bit fixed point DSP (TMS320c50) is used for controlling the SR motor. With the help of DSP, the sampling and execution time achieved for the whole process is $100 \mu s$, which is quite satisfactory for this applications. The algorithm is tested on a $4 \mathrm{~kW}, 4$-phase, $8 / 6$ pole OULTON motor. A split-capacitor link power converter is used for controlling the motor. The schematic of the power converter is shown in Fig. 6 .

\section{EXPERIMENTAL RESULTS}

With the above estimation process and TI DSP, the motor is run truly sensorless. It has been possible to obtain position estimation with errors less than $\pm 1.5^{\circ}$ for the $8 / 6 \mathrm{SR}$ motor at speeds upto $1500 \mathrm{rpm}$. In order to illustrate the accuracy of the proposed method, estimated position is compared with the actual position obtained from a position sensor. A typical test result at $1500 \mathrm{rpm}$ is shown in Fig. 7. The motor can start smoothly from any arbitrary initial position following the proposed method. Figure 8 illustrates the position estimation results and phase current of a particular phase during starting. Further, the speed and phase current trajectories with and without position sensors are shown in Fig. 9. Similarly, the speed transient from standstill to rated speed $(1500 \mathrm{rpm}$ in this case) with estimated position and with position sensor are plotted in Fig. 10. The test results confirm that the proposed estimator may be used in practical applications without sacrificing the performance of the drive.

\section{CONCLUSION}

The discussion and test results in the preceding sections lead to the conclusion that truly sensorless operation of SR motor in the entire speed range is possible. Starting ambiguity is avoided by the proposed estimator and accuracy

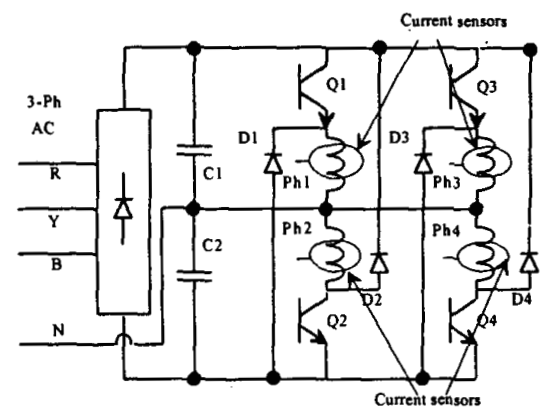

Fig. 6 Converter for SR motor drive

of estimation is improved with appropriate corrections for mutual flux and eddy current. TI DSP with its sophisticated architecture helps realising the controller in real time.

\section{REFERENCES}

1. W.F. Ray, I.H. Bahadly, "Sensorless Methods for Determining the Rotor Position of Switched Reluctance Motor", Proc. of 5th Europeon Conference on Power electronics and Applications, IEE publication no. 377, Sept. 93, Vol. 6, pp 7-13.

2. Debiprasad Panda and V. Ramanarayanan, "Effect of Mutual Inductance on Steady-state Performance and Position Estimation of Switched Reluctance Motor Drive", Published in the Proceedings of IEEE Industry Application Society Annual Meeting, 1999.

3. Debiprasad Panda and V. Ramanarayanan, "An Accurate Position Estimation Method for Switched Reluctance Motor Drive", International Conference on Power Electronics Drives and Energy Systems (PEDES'98), Perth, Australia, December, 1998, pp 523-528.

4. V. Ramanarayanan, L. Venkatesha, Debiprasad Panda, "Flux-linkage Characteristic of Switched Reluctance Motor", Power Electronics Drives and Energy System conference( PEDES-96 ) Delhi, December 1996, pp 281-285.

5. G. G. Lopez, P. C. Kzaer, T. J. E. Miller, "A New Sensorless Method for Switched Reluctance Motor Drives", IEEE Transaction on Industry Applications, Vol. 34, No.4, July/August, 1998, pp. 832-840.

6. Miller T. J. E.,"Switched Reluctance Motors and Their Control", Magna Physics Publishing And Clarendon Press Oxford 1993.

7. G. G. 'Lopez, P. C. Kzaer, T. J. E. Miller, "High-grade Position Estimation for SRM Drives using Fluxlinkage/Current Correction Model", IEEE IAS Conferrence, 1998.

8. Jianrong $\mathrm{Bu}$ and Longya $\mathrm{Xu}$, "Eliminating Starting Hesitation for Reliable Sensorless Control of Switched Reluctance Motors", IEEE IAS Annual meeting, 1998. 
(i)

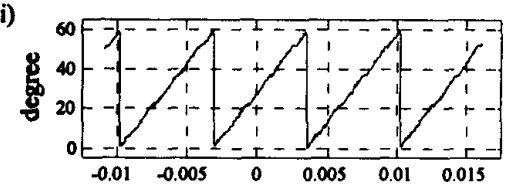

(ii)

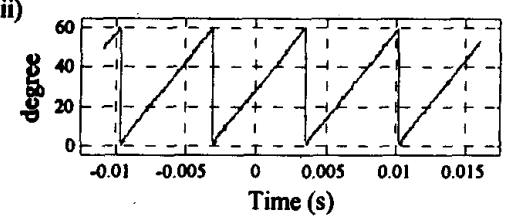

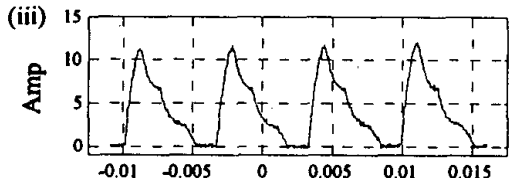

(i)

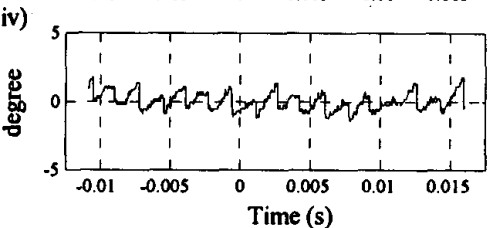

Fig. 7 Experimental results of (i) Estimated position, (ii) Actual position, (iii) Phase current and (iv) Net error in estimation (Difference between Actual Posn and Estimated Posn) at $1500 \mathrm{rpm}$ and 10Nm load.

[Vph $=220 \mathrm{~V}$ and ; Trace (iii) and (iv) are not synchronised with Trace (i) and (ii)]

(a)

(i)

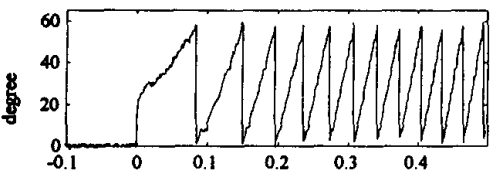

(ii)

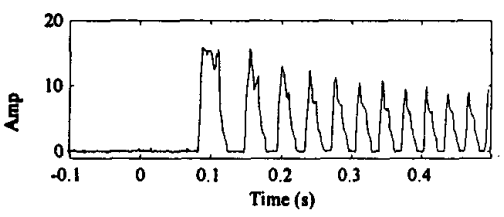

(b)

(i)

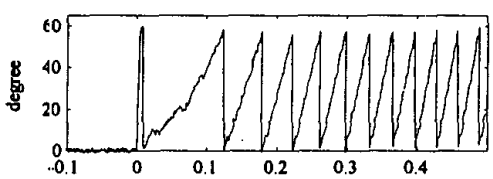

(ii)

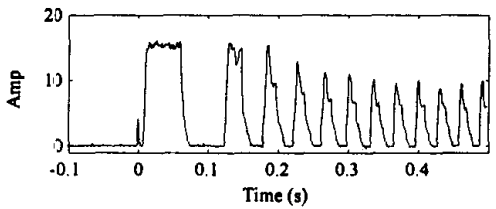

Fig. 8 Experimental result during start without position sensors: (i) Estimated position and (ii) Phasel current -(a) with initial position of $30^{\circ}$ and (b) with initial position of $55^{\circ}$.

(a)

(i)

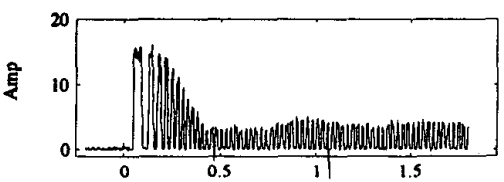

(ii)

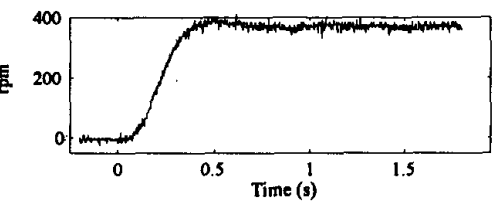

(b)

(i)

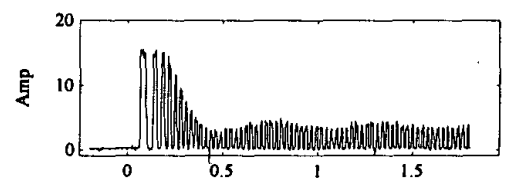

(ii)

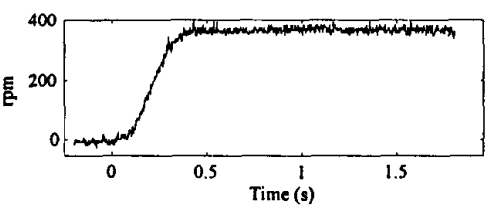

Fig. 9 Experimental result of (i) Phase 1 current and (ii) Speed waveform during start (0 to $375 \mathrm{rpm}$ ) -(a) with position sensors, (b) without position sensors.

(i)

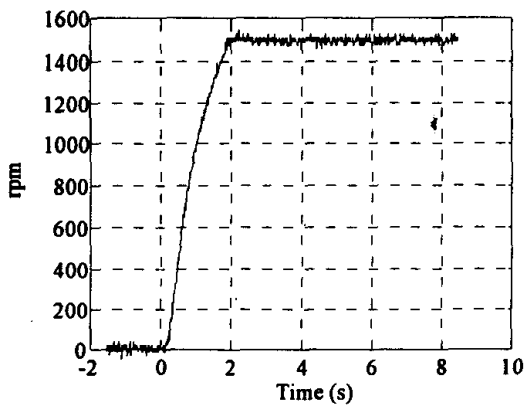

(ii)

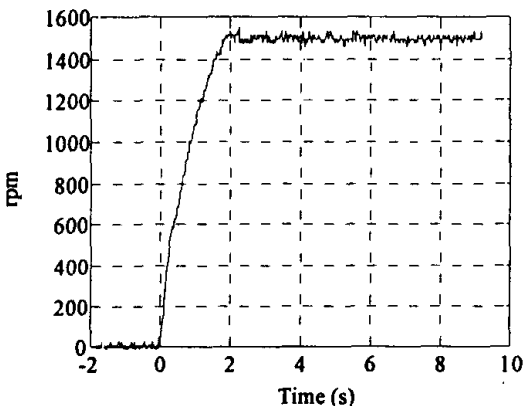

Fig. 10 Test results of Speed Transient: (i) with estimated position, (ii) with position sensor from 0 to $1500 \mathrm{rpm}$ 